\title{
Médiévales
}

Langues, Textes, Histoire

57 | automne 2009

Langages politiques, $\mathrm{XII}{ }^{\mathrm{e}}-\mathrm{XV}^{\mathrm{e}}$ siècle

\section{Droit canon et littérature chevaleresque : l'image du rex inutilis dans le roman de Cleriadus et Meliadice}

Canon Law and Chivalric Literature: the Image of the Rex Inutilis in the Cleriadus et Meliadice Romance

\section{Lidia Amor}

\section{OpenEdition}

\section{Journals}

Édition électronique

URL : https://journals.openedition.org/medievales/5811

DOI : 10.4000/medievales.5811

ISSN : 1777-5892

\section{Éditeur}

Presses universitaires de Vincennes

Édition imprimée

Date de publication : 20 décembre 2009

Pagination : 137-150

ISBN : 978-2-84292-241-2

ISSN : 0751-2708

\section{Référence électronique}

Lidia Amor, «Droit canon et littérature chevaleresque : l'image du rex inutilis dans le roman de Cleriadus et Meliadice », Médiévales [En ligne], 57 | automne 2009, mis en ligne le 19 janvier 2012, consulté le 23 avril 2022. URL : http://journals.openedition.org/medievales/5811 ; DOI : https://doi.org/10.4000/ medievales.5811 
Médiévales 57, automne 2009, p. 137-150

Lidia AMOR

\section{DROIT CANON ET LITTÉRATURE CHEVALERESQUE : L'IMAGE DU REX INUTILIS \\ DANS LE ROMAN DE CLERIADUS ET MELIADICE}

\section{La littérature chevaleresque à la cour de Philippe le Bon}

Le patrimoine littéraire du milieu bourguignon est un exemple de l'activité artistique et intellectuelle foisonnante qui s'épanouit à la cour des ducs de la maison de Valois pendant le $\mathrm{Xv}^{\mathrm{e}}$ siècle. Il s'agit d'un domaine de recherche idéal pour comprendre cette période de transition, pendant laquelle certains paramètres sociaux et culturels médiévaux et ceux qui caractériseront la Renaissance convergent vers une synthèse enrichissante.

Cleriadus et Meliadice, roman chevaleresque de la première moitié $\mathrm{du} \mathrm{XV}^{\mathrm{e}}$ siècle, constitue l'un des phénomènes littéraires qui illustre le mieux cette idée et qui fournit des renseignements intéressants pour apprécier la culture de l'époque. Pourtant, en dépit de la popularité attestée par les traditions manuscrite et imprimée ${ }^{1}$, il est resté inaperçu des médiévistes à

1. Les manuscrits conservés sont: Tours, Bibliothèque Municipale 952, ms. sur papier de 325 ff. ; Bruxelles, Bibliothèque Royale, IV 1002, ms. sur papier de 164 ff. ; Chantilly, Musée Condé 650, ms. sur parchemin, de 128 ff. ; Londres, British Library, B.M. Royale 20 CII, ms. sur parchemin de 236 ff. ; P1 Paris, BnF, franç. 1439 ms. sur papier de 371 ff. ; P2 Paris, BnF, franç. 1440, ms. sur papier de 287 ff. et P3, Paris, BnF, franç. 1494-1495 ms. sur papier, écrit à pleines lignes, réparti en deux volumes de 113 et $201 \mathrm{ff}$; Turin, Biblioteca Nazionale dell'Università 1628 L.II.2, ms. sur parchemin de 99 ff. ; Vienne, Österreichische Nationalbibliothek 3427, ms. sur papier de $160 \mathrm{ff}$. et Leipzig, Universitätsbibliothek, II.Rep.109. Les imprimés sont : Vér, première édition gothique due à Antoine Vérard, 1495 in-fol. sur vélin de 95 p. ; Len1, édition gothique de Michel Lenoir, in $-4^{\circ}$ de 199 p. connue par deux exemplaires (Paris, BnF, Rés. Y² 680 ; Chantilly, Musée Condé III F 105 (la division du texte en un Prologue et 39 chapitres servira de modèle à toutes les éditions postérieures); Len2, nouvelle édition gothique de Michel Lenoir, in-4 $4^{\circ}$ de 197 p. connue par deux exemplaires (Paris, BnF, Rés. Y² 676, lacunaire ; Londres, British Library C48 e II, dont les paginations ne concordent pas) ; Serg, édition gothique de Pierre Sergent, in $-4^{\circ}$ de 200 p. connue par trois exemplaires (Paris, BnF, Rés. Y² 677 ; Nantes, Musée Dobrée 553 ; Édimbourg, National Library of Scotland, H. 23 ca 3) et Arn, édition gothique d'Olivier Arnoullet de 1529, in- $4^{\circ}$ de $211 \mathrm{p}$. dont il ne reste qu'un exemplaire (Paris, Bibliothèque de l'Arsenal, Rés. $4^{\circ}$ B. L. 4280, ancien fonds La Vallière $n^{\circ} 4025$ ). 
l'exception des brefs commentaires de Georges Doutrepont ${ }^{2}$, des examens de son éditeur moderne, Gaston Zink ${ }^{3}$, et d'une série d'articles qui étudient différents aspects thématiques du roman ${ }^{4}$.

Cleriadus et Meliadice raconte la biographie de Cleriadus, fils du comte des Asturies, depuis sa jeunesse jusqu'à son mariage avec Meliadice. Il accompagne son père (désigné comme administrateur du royaume par le roi Phellipon) en Angleterre, où il déploiera ses atouts physiques et moraux dans des aventures, des tournois, des joutes et dans la guerre contre les infidèles à Chypre, faits d'armes qui lui valent le titre de meilleur chevalier du monde. L'histoire de ses exploits chevaleresques s'entrelace avec celle de ses amours avec la princesse anglaise, Meliadice. Toutefois, leur passion sera assombrie par le réquisitoire de Thomas de l'Angarde, demi-frère du roi Phellipon. En effet, Thomas accuse Meliadice et Cleriadus de régicide, dénonciation qui est à l'origine de l'exil de la jeune fille et de l'usurpation du pouvoir de la part de Thomas. Cette dernière action sera, par la suite, désamorcée à travers le siège mené par Cleriadus dans le but de rétablir l'ordre ancien. Ainsi, le malheur cerne les amants : Meliadice, condamnée à mort, se sauve grâce à la pitié de ses bourreaux, quoique ses souffrances ne s'achèvent qu'au moment où elle rencontre Cleriadus aux Asturies. Après leur réunion, les jeunes gens retournent en Angleterre, passant d'abord par le royaume de France, où le roi les accueille formidablement. Lors de leur arrivée en Angleterre, Phellipon, conscient de ses erreurs, demande le pardon des jeunes gens, abdique en faveur de Meliadice et l'offre en mariage à Cleriadus. L'histoire finit avec une brève description du gouvernement du roi Cleriadus et de la reine Meliadice et de leur successeurs.

Le canevas de l'amour entre les jeunes gens et celui des mésaventures de Meliadice révèlent l'influence dans la composition de Cleriadus et Meliadice de deux types littéraires spécifiques : le roman idyllique et les récits (épiques et romanesques) assemblés autour du motif de la dame injustement accusée.

2. G. Doutrepont, La littérature française à la cour de Bourgogne, Genève, 1970.

3. Cleriadus et Meliadice, éd. G. Zink, Genève, 1984, p. IX-XXXII.

4. Il faudrait mentionner les articles suivants : C. GALDERISI, «Clériadus et Méliadice. Une chronique de motifs littéraires du Moyen Âge », Vives Lettres, 1999, p. 85-97 ; M. SzKILNIK, «A Pacifist Utopia, Clériadus et Méliadice», dans Inscribing, the Hundred Years'War in French and English Cultures, D. BAKER éd., Albany, 2000, p. 221-235 ; D. BOHLER, « Péninsule ibérique et îles de Bretagne : la géopolitique de l'imaginaire romanesque au $\mathrm{XV}^{\mathrm{e}}$ siècle », dans E. BURY et F. MoRa dir., Du roman courtois au roman baroque, Paris, 2004, p. 279-293 ; C. Gaullier-Bougassas, "La croisade dans le roman chevaleresque du $\mathrm{XV}^{\mathrm{e}}$ siècle », Ibid., p. 295-307 ; C. ROLLIER-PAULIAN, "L'errance du couple noble : évolution d'un outil didactique dans le roman du XIV e au XV e siècle (l'exemple de Clériadus et Méliadice) », Ibid., p. 267-277; L. AMOR, «Diálogos textuales : una comparación entre Ponthus et Sidoine y Cleriadus et Meliadice ", Fifteenth Century Studies, 33, 2008, p. 55-73; L. Amor, "El Roman de Clériadus et Méliadice : transformaciones de un paradigma heroico y su vinculación con el poder», dans N. Guglielmi et A. RucQuor dir., Derecho y justicia : el poder en la Europa medieval. Droit et justice : le pouvoir dans l'Europe médiévale, Buenos Aires, 2008, p. 215-246. 
Or, si la thématique développée dans ces épisodes montre surtout des rapports avec la littérature romanesque, il n'en reste pas moins qu'elle exprime une certaine appartenance du roman à la littérature édifiante, notamment en ce qui concerne les attributs et la conduite souhaités chez le prince idéal, préoccupations majeures des juristes, philosophes et fonctionnaires royaux de toutes les cours européennes durant les $\mathrm{XIV}^{\mathrm{e}}$ et $\mathrm{XV}^{\mathrm{e}}$ siècles.

Dans cet article, notre intention sera de montrer que non seulement l'exil de Meliadice rapproche l'œuvre de la tradition romanesque des temps passés mais aussi que l'entrelacement du récit du bannissement avec celui de la guerre à Chypre (chapitres XXI à XXIV) laisse percevoir une réflexion sur le rex inutilis, figure présente dans le droit canonique. De telle sorte, la doctrine sur le rex inutilis devient le matériau de la fiction et engendre un réseau mnémotechnique chez le lecteur où histoire, jurisprudence et littérature se rejoignent. Nous démontrerons qu'en utilisant le motif de la dame injustement accusée, l'auteur évoque certains romans et propose à l'auditoire une lecture politique de Cleriadus et Meliadice. Ensuite, nous analyserons la façon dont le roi Phellipon, emblème de fragilité, correspond à la figure d'Henri II de Lusignan (1285-1306 et 1310-1324) pendant les événements qui bouleversèrent son règne et auxquels l'auteur de Cleriadus et Meliadice fait allusion par le biais du récit de la guerre sainte à Chypre.

\section{La faiblesse royale et sa manifestation dans les histoires de reines injustement accusées}

L'exil de Meliadice, résultat de la miséricorde qu'elle éveille chez ses bourreaux, est un dénouement plus heureux que celui que son père avait envisagé pour elle, c'est-à-dire la peine de mort. Les mésaventures que la princesse devra surmonter établissent un rapport entre ce roman et, comme nous l'avons déjà indiqué, un ensemble de textes réunis autour du motif de la dame injustement accusée. L'inculpation - en général à travers l'introduction de fausses lettres, mais aussi comme résultat des mensonges d'un envieux ${ }^{5}$ - s'inscrit, en outre, dans le récit paradigmatique de l'impératrice de Rome, qui a été très productif dans la littérature française depuis le $\mathrm{XIII}^{\mathrm{e}}$ siècle et qui a encouragé des lectures historiques et sociologiques chez les médiévistes, parmi lesquelles on distingue la perspective juridique signalée par Nancy Black:

5. La fausse inculpation se rapporte, en général, à l'adultère de la femme. Ce motif apparaît aussi dans Le Roman de la Rose ou de Guillaume de Dole (représentatif - d'après la définition de Gaston Paris - du Cycle de la Gageure, cf. Romania XXXII, 1903). Par ailleurs, l'Histoire des Seigneurs de Gavre, R. STUIP éd., Genève, 1993, peut aussi être considérée comme une variante de ce motif puisque le conflit détermine l'errance de Louis de Gavre, déclenchée avant sa naissance, lorsque sa mère est accusée d'adultère par son mari. Bien que ces histoires introduisent ce motif, leur développement narratif diffère de celui qu'on trouve dans Cleriadus et Meliadice. 
Narratives involving slander and forged documents would have been of particular interest to government officials, lawyers, and members of both the bourgeoisie and the nobility.

[...] forged documents undermine the legal validity usually granted written and signed documents. Therefore, the story of falsely accused noblewoman can also be read as a case study in judicial injustice.

The happy endings - when her innocence is proved, when the false slanderers are exposed, and when she is reunited with her family - are a kind of plea for trust in the judicial system, however senseless or unfair it may be at times ${ }^{6}$.

Dans la littérature française médiévale, l'inculpation arbitraire de la femme est un motif qui apparaît dans plusieurs textes, parmi lesquels nous pouvons citer La fille du comte de Ponthieu ${ }^{7}$, datée du XIII ${ }^{\mathrm{e}}$ siècle, œuvre qui, au $\mathrm{XV}^{\mathrm{e}}$ siècle, constitue un cycle en prose avec le Dit du prunier ${ }^{8}$ (source de Jehan d'Avennes) et le Roman de Saladin ${ }^{9}$. Il existe, en outre, une variante textuelle qui s'enchaîne avec le motif de l'inceste (couramment entre père et fille) diffusée dans l'Europe médiévale au moyen de l'histoire d'Apollonius de $\operatorname{Tyr}^{10}$ et présent également dans La Manekine de Phillipe de Rémi ${ }^{11}$, le Roman du Comte d'Anjou de Jehan Maillart ${ }^{12}$ et La Belle Hélène de Constantinople $^{13}$. Dans ces romans, le motif de l'inceste et celui de la femme accusée injustement mettent au premier plan le personnage féminin et permettent le dialogue entre le discours romanesque et l'hagiographie ${ }^{14}$. Il n'en reste pas moins que Meliadice gagne une place principale dans cette biographie chevaleresque grâce à l'accusation dont elle fait l'objet. Cette circonstance assure l'impact du groupe textuel cité plus haut sur Cleriadus

6. N. Black, Medieval narratives of accused queens, Gainesville, 2003, p. 10.

7. La hija del conde de Ponthieu. La fille du Comte de Ponthieu, E. CoBos CASTro éd., Barcelone, 1988.

8. Le dit du Prunier. Conte moral du Moyen Âge, P. BADEL éd., Genève, 1985.

9. D. Quéruel (Splendeurs de la cour de Bourgogne, D. RÉGNIER-BOHLER éd., Paris, 1995, p. 371-2) explique qu' « un lien généalogique, entièrement fictif, a été imaginé par l'auteur $\mathrm{du} \mathrm{XV}^{\mathrm{e}}$ siècle, afin de réunir étroitement les trois récits. Tout est fait pour établir entre Jean d'Avesnes, seigneur de Hainaut devenu par son mariage comte d'Artois, la fille du comte de Ponthieu et Saladin, un lignage illustre et inattendu : chapitres de transition, rubriques, insertion de noms propres contribuent à faire de cet ensemble une composition cyclique. » De même, l'auteur signale qu'il n'y a que deux manuscrits qui conservent ce cycle d'origine nettement bourguignonne : l'un appartenait à la famille Croÿ et l'autre est inventorié vers 1468 dans la bibliothèque de Philippe le Bon. Cf. D. QuÉRUEL, «Introduction», L'istoire de tres vaillans princez monseigneur Jehan d'Avennes, Villeneuve d'Ascq, 1997.

10. Le roman d'Apollonius de Tyr, M. ZINK éd., Paris, 1982.

11. Le roman de la Manekine (Paris, BnF fr. 1588), B. SARgent-BAur éd., AmsterdamAtlanta, 1999.

12. Jehan Maillart, Le roman du Comte d'Anjou, M. Roques éd., Paris, 1974.

13. La Belle Hélène de Constantinople : chanson de geste du XIV e siècle, C. Roussel éd., Genève, 1995.

14. N. Black, Medieval narratives..., op. cit., p. 12 affirme : «the popularity of these narratives can be explained by their close relationship to biblical stories, hagiographic texts, and other narratives of abused women, such as those told by Chaucer in his Legend of Good Women. » 
et Meliadice, si bien que le rôle central de Meliadice n'est pas une conséquence de la diversification de ses (més)aventures mais le résultat du choix de l'auteur qui a réuni le motif de l'injuste inculpation de la femme, le siège de Londres mené par Cleriadus et la guerre à Chypre. Par ailleurs, l'expérience effrayante de la jeune fille dans la forêt - après avoir été libérée par ses bourreaux - rappelle au lecteur Berthe as grans piés ${ }^{15}$. En effet, Berthe, comme Meliadice, accusée à tort et condamnée à mort, doit rôder dans des espaces épouvantables avant de recouvrer sa condition royale. Il faut toutefois reconnaître qu'à l'exception de cet épisode, le récit de Berthe as grans piés diffère notablement de celui de Cleriadus et Meliadice. Berthe, victime d'une mystification, est dépouillée de son identité sociale : elle n'est pas la destinataire directe de la tromperie mais elle souffre, en tout premier lieu, de l'avarice de Margiste, sa nourrice.

Ce bref parcours, par la littérature, des femmes calomniées met en évidence la lecture politique que les auteurs des romans paraissent proposer à l'auditoire, selon laquelle les souffrances féminines sont le corollaire de l'impuissance des souverains, d'autant plus que les femmes inculpées ne méritent pas les tribulations qu'elles doivent surmonter.

\section{Camino paupertatis : la purification du royaume}

La fausse lettre censée découvrir le projet d'assassiner Phellipon de la part de Meliadice et Cleriadus scelle non seulement le destin de la princesse et du chevalier mais aussi celui de l'Angleterre. Thomas installe une crise fictive qui, en fait, découvre le besoin d'un changement dynastique imminent. On pourrait considérer que la conduite du roi Phellipon et la trahison de Thomas expliquent à elles seules la visée politique et sociale du roman. Pourtant, comme nous venons de le signaler, le motif de la dame injustement accusée contribue à ce dessein politique en même temps qu'il en fournit les solutions possibles en favorisant un mariage exogamique, représenté ici par l'union de la princesse et du chevalier.

Après la disparition de Meliadice, Cleriadus doit recomposer l'ancien ordre anglais, objectif atteint à travers la sédition contre Thomas - administrateur de facto de Phellipon. Néanmoins, il ne peut pas restituer l'élément principal de l'harmonie sociale : Meliadice. L'exil volontaire du jeune chevalier manifeste aussi bien la grandeur de sa passion que l'état de pénurie du royaume, du moment que les Anglais ont perdu l'objet (Meliadice) qui rendrait possibles des alliances avec d'autres royaumes. En outre, les lamentations des courtisans anglais redoublent puisqu'ils sont dépouillés et de l'héritière et du successeur au trône (Cleriadus). L'avenir du pays est à tel point enchaîné au destin de la princesse que la détresse que les seigneurs 
anglais devraient supporter est comparée à celle de Meliadice. En effet, les souffrances de la jeune fille représentent le chemin de purification que le roi et ses barons devraient parcourir de manière à réparer le dommage infligé au peuple en permettant la mort de la princesse.

Or, l'hagiographie avait déjà adopté la métaphore du salut du peuple en raison de la douleur du saint ou de la sainte, sentiment caractérisant, tout d'abord, la passion du Christ. Ce n'est pas un hasard si le narrateur a recours à ce thème lorsque Meliadice fait sa prière au milieu de la forêt ${ }^{16}$ :

- He ! Mon tresdoulx Dieu, je sçay et croy fermement que, par vostre grant humilité, volutes prandre char en la benoiste Vierge Marie et d'elle naquistes virginellement et sans douleur avoir et aussi volustes prandre mort et passion pour tout humain lignaige racheter, en l'arbre de la Croix, et, au tiers jour, ressuscitates et toutes peines et douleurs volustes souffrir pour nous. Et, en l'onneur de vostre digne Passion, sire, vueil je souffrir toutes peines et pouvreté, car tant ne savroye faire pour vous que vous avez fait pour moy. Hellas ! sire, moy qui suis une pouvre pecharesse et qui n'ay pas congneu les grans biens que faiz m'avez, dont, sire, vous crye mercy humblement en vous requerant vrayement, comme vous pouez toutes choses faire et comme vous savez que en cecy je n'ay coulpe, que vous me vueillez aider et conforter en ce besoing et metz mon corps et mon ame et tout mon fait en vostre digne main ${ }^{17}$.

Contrairement aux saintes, le lien entre Meliadice et les seigneurs signale non seulement l'abnégation de la princesse mais aussi son rôle en tant qu'objet-sujet du bonheur anglais. Meliadice est le sauf-conduit des courtisans : elle représente l'espérance de paix sociale et le camino paupertatis qu'elle doit parcourir est celui du roi et de ses conseillers. Autrement dit, le narrateur dresse un portrait de la noblesse et du roi anglais qui montre leur anémie politique, et cette image devient encore plus négative lorsque l'on confronte la description de l'armée française et anglaise pendant la guerre à Chypre contre les infidèles : tandis que le roi de France envoie son connétable à la tête de ses hommes, Phellipon doit désigner comme chef de son armée un étranger, Cleriadus. Ces scènes, disseminées dans le continuum narratif, s'actualisent dans la mémoire du lecteur au moment où sont racontés les malheurs de la jeune fille.

Si les expériences affreuses de Meliadice en exil servent à justifier son influence sur la destinée politique du royaume anglais, le comportement de son père, Phellipon, révèle son manque de prudence et de puissance

16. Il est possible d'interpréter en ce sens la reconnaissance de Meliadice envers ses bourreaux, auxquels elle donne ses vêtements : «- Beaulx seigneurs et mes tresdoulx amis, je ne vous ay que donner, se ce n'est ma cotte simple que je vous donne et ceste chenecte d'or que j'ay en mon coul. [...] Lors elle la prent et la mect hors de son coul où il pendoit ung moult riche fermillet et puis despoulle sa cotte simple et leur baille tout et la pouvre fille demeure toute nue en sa cheminse. » (chap. XXIII, p. 302-303).

17. Chap. XXIII, p. 300. 
puisqu'il croit naïvement aux mensonges de son demi-frère. En ce sens, le chapitre XXVI de Cleriadus et Meliadice montre, comme s'il s'agissait d'un exemplum, la débâcle qui s'abat sur un royaume quand le pouvoir est exercé par un souverain faible, tel que le narrateur l'annonce à travers l'une de ses rares interventions :

Et, pour ce, a icy bonne exemple comment nul roy ne prince ne doit croire legerement, pour quelque rapport que on lui face, et aussi de faire hastivement justice s'il veult ouvrer sagement. Cleriadus relieve le roy en son estant et lui dist :

- Sire, à moy ne devez pas requerre pardon, mais à Dieu premier envers qui vous avez moult failly et puis à tous ceulx de vostre royaume. Or pensez et advisez, ainsi que je vous prie, de faire justice de ce traistre, car vous vées clerement la grant traïson que il a faicte, comme il recongnoist de sa bouche ${ }^{18}$.

Dans Cleriadus et Meliadice, ceux qui exercent le pouvoir sont caractérisés selon des angles différents - bon gouvernant, tyran, roi débile - et de façon à ce que le lecteur puisse d'abord reconnaître l'essence de chaque modèle ${ }^{19}$ et ensuite relier les idées qui ont circulé concernant la figure du prince aussi bien dans le domaine universitaire que dans les circuits nobiliaires, en particulier bourguignons. De cette sorte, Cleriadus et Meliadice rejoint la mentalité $\mathrm{du} \mathrm{XV}^{\mathrm{e}}$ siècle, comme l'exemplum ${ }^{20}$ est associé au sermon. Ainsi, le roman s'articule avec la littérature édifiante et l'histoire factuelle comme s'il était un exemplum encadré dans une textualité majeure et met en évidence la façon dont le savoir circulait à la fin du Moyen Âge. Il raconte une biographie chevaleresque dans laquelle fiction littéraire, droit, philosophie et histoire factuelle constituent un réseau sémantique qui dévoile une riche perception de la réalité européenne contemporaine. Finalement, le roman bourguignon démontrerait que l'Histoire est un texte créé à partir d'autres textualités provenant de divers domaines de la culture et formée par un réseau des signifiés découlant d'une mentalité spécifique (c'est-à-dire une noblesse à la recherche d'une identité sociale rajeunie) avant l'affermissement de la monarchie centrale.

18. Chap. XXVI, p. 359.

19. Il manifeste la fonction publicitaire du texte tel que B. GUENÉE, L'Occident aux XIV et $X V^{e}$ siècle, Paris, 1998, p. 94, l'avait définie : «Les publicistes répandent ainsi des opinions qui sont souvent la traduction politique d'une réflexion philosophique. »

20. D'après Cl. Bremond et J. Le GoFF, Typologie des sources du Moyen Âge occidental : l'exemplum, Turnhout, 1982, p. 27-42, l'exemplum ne désigne pas un homme mais un récit. Il s'agit d'une histoire considérée comme instrument didactique, subordonnée à un texte qui l'enserre ; il n'est pas son ornement mais un élément essentiel. Or, selon l'opinion des historiens, son insertion dans un texte majeur suppose la technique du collage, de la même manière que l'auteur de Cleriadus et Meliadice compose le roman. Cette idée pourrait expliquer l'une des possibles dérivations des rapports entre histoire et littérature. 
En dernier lieu, il faudrait dire que la trahison de Thomas de l'Engarde est porteuse d'une double fonction : d'une part elle s'accommode de la structure linéaire du récit, d'autre part, elle fait ressortir la perspective moralisante de cette biographie chevaleresque, consacrée à dresser le portrait exemplaire d'un homme destiné à la dignité royale. En conséquence, Cleriadus et Meliadice instaure un univers significatif régi par la littérature romanesque, par la littérature édifiante et par les idées sur le prince idéal que les intellectuels diffusaient aux $\mathrm{XIV}^{\mathrm{e}}$ et $\mathrm{XV}^{\mathrm{e}}$ siècles. Il est possible, alors, de reconstruire ce circuit d'autant plus que ce roman reprend la forme et la fonction des exempla.

\section{Du roi débile de la fiction au rex inutilis du droit canonique : convergences}

L'incapacité de Phellipon met en danger le destin des Anglais dès le début de l'histoire, c'est pourquoi il sollicite le soutien du comte des Asturies comme administrateur du royaume. Cleriadus doit toujours défendre les intérêts du pays et Palixés et Amador - les cousins et les lieutenants de Cleriadus - doivent protéger la nation pendant l'absence du jeune héros. Ainsi, Phellipon réussit à maintenir l'ordre dans le pays grâce à la prudence du comte des Asturies et à l'intervention constante de Cleriadus pour défendre les territoires anglais ${ }^{21}$. L'impuissance royale exige un changement dynastique imminent, action qui détermine, d'ailleurs, le caractère central de Meliadice : la princesse est contrainte de se marier avec un homme illustre, lequel assure non seulement la continuité du lignage mais en plus garantit, moyennant son pouvoir et son autorité, le bonheur du pays. Quoique Meliadice soit la seule héritière de Phellipon, ses prétentions au trône s'effacent devant celles de son oncle Thomas qui, par droit de succession, peut aussi recevoir la couronne. Qui plus est, l'histoire factuelle et le droit public légitiment les aspirations de Thomas plutôt que celles de Meliadice, circonstance qui fait basculer la possibilité de descendance directe de Phellipon.

D'après la Loi Salique ${ }^{22}$, Thomas - et non pas Meliadice ou son époux - devrait être sacré roi et exercer, légitimement, le pouvoir royal.

21. En ce sens, Cleriadus et son père ne sont pas semblables. Le père se consacre à la préservation de ses intérêts et ceux de sa communauté tandis que le fils doit intervenir chaque fois que la crise resurgit en Angleterre.

22. J. KRYNEN, L'empire du roi : idées et croyances politiques en France, XIII ${ }^{e}-X V^{e}$ siècle, Paris, 1993, p. 133-134, affirme que « [...] lorsque Jean de Vignay traduit, entre 1337 et 1350 , les fameux Échecs moralisés (de Jacques de Cessoles), il évoque, s'agissant de la reine, la "constitution" faite "moult de temps avant Charlemagne [...] gardée par tous les rois depuis icelui temps". C'est bien la loi salique qui est visée, et le traducteur sait qu'un tel texte peut être utilisé pour la succession du royaume. Vers 1358, la découverte par l'historiographe de France, Richard Lescot, du texte de la loi salique n'est donc pas fortuite, pas plus que son interpolation du titre $D e$ allode faisant accroire la rédaction suivante: Mulier vero nullam in regno habeas portionem [...]. L'imprécision avec laquelle les principaux conseillers de Charles V avancent l'existence d'une loi écrite signifie peut-être qu'ils n'ont pas connu ou pas su tirer parti de la trouvaille et de l'interprétation de Richard Lescot. Il reviendra à Jean de Montreuil, sous le règne suivant, de donner son véritable départ au mythe de la loi salique. » 
Dans le contexte social et culturel des $\mathrm{XIV}^{\mathrm{e}}$ et $\mathrm{XV}^{\mathrm{e}}$ siècles, aucune exception ne pouvait empêcher son arrivée au trône, sauf si Thomas démontre qu'il n'est pas compétent pour cette mission, circonstance dévoilée au lecteur au tout début du roman, lors de la description du personnage ${ }^{23}$ :

Or avoit le roy ung frere, beau chevalier et jeune, de l'aaige de trante ans ou environ, mais pour nulle riens ne lui eust baillé le roy le gouvernement de son royaume, car cellui seigneur estoit plain de deshonnestes taches comme estre fel et orgueilleux, plain de ire, cruel en toutes choses et irraisonnable, pourquoy le roy, son frere, ne lui vouloit pas bailler le gouvernement de son royaume et aussi à nul des autres seigneurs ${ }^{24}[\ldots]$.

Thomas possède les défauts les plus inquiétants que l'on puisse imaginer chez un monarque; on découvre son inaptitude pendant la tyrannie qu'il exerce sur les Anglais après avoir trompé Phellipon et avoir réussi à éloigner Meliadice et la famille de Cleriadus de la cour. Depuis cette perspective, la trahison amplifie la faiblesse de l'ancien roi et elle oppose deux types de gouvernement : le bon gouvernement et la tyrannie. Les diverses manières d'exercer le pouvoir sont comparées afin que le lecteur puisse comprendre, grâce au raisonnement dialectique, les avantages et les désavantages induits par les diverses prémisses juridiques.

L'accession au trône d'une femme ou d'un membre masculin de la famille royale descendant d'une branche féminine fut automatiquement exclue après l'imposition de la Loi Salique qui ébranla les desseins successoraux d'Édouard III à la couronne de France à la mort de Charles le Bel. En ce sens, Cleriadus et Meliadice se présente comme une sorte de cas d'école: l'auteur expose le besoin d'un renouvellement dynastique à cause de la faiblesse royale et le seul héritier direct au trône est une femme ; il existe, par ailleurs, un héritier masculin mais ses vices lui interdisent d'y accéder. Ces données permettent au lecteur de réfléchir sur les contradictions provoquées par la confrontation de deux principes juridiques distincts. En outre, la situation devient plus complexe puisque le souverain anglais, conscient de son impuissance, avait déjà nommé le comte des Asturies administrateur du royaume, l'autorité avait donc été déléguée au comte tandis que Phellipon conservait toujours la dignitas regia. Cette nomination signale que la figure du rex inutilis se dressait déjà au début du roman.

Les fausses lettres servent à augmenter l'incapacité de Phellipon et, en même temps, dépeignent le traître comme un usurpateur, puisque son

23. Il faudrait dire que Cleriadus arrive au trône irlandais à travers sa lignée maternelle. Cette façon d'y accéder reprend une coutume antérieure à l'imposition de la Loi Salique. De telle sorte, on donne à cette loi une temporalité puisqu'elle suppose une nouveauté par rapport au droit coutumier et correspond au besoin d'une loi positive qui s'accommode davantage avec les lois naturelles et divines dans une situation historique précise.

24. Chap. I, p. 2-3. 
action entraîne le renversement de celui qui exerçait effectivement l'autorité en Angleterre, c'est-à-dire, le comte des Asturies :

[...] messire Thomas, son frere, l'a mené [a Phellipon], lequel le gouverne tout ainsi que il lui plaist. Et, sire, il n'est demouré officier que le roy $\mathbf{y}$ ayt mis, ne monseigneur vostre pere, que messire Thomas ne ayt osté et y en a remis d'autres à son voulenté et moy mesmes ne suis je plus maire de ceste ville. Mais encores y a plus, qui est dommaige inreparable. C'est que messire Thomas, par sa grant mauvaistié et faulce traïson $\left[162 \mathrm{v}^{\circ}\right]$ et par son mauvais rapport qu'il a fait au roy, dont le roy a esté indigné et mortellement courroucé qu'il a fait mourir madame Meliadice ${ }^{25}$.

Par ailleurs, le comte des Asturies réussit à sauvegarder le statu quo de la nation, mais c'est Cleriadus qui devra la défendre contre les crises extérieures. Évidemment, Phellipon avait prévu la solution des difficultés immédiates mais il ne pouvait pas garantir l'avenir de l'Angleterre, puisqu'il n'avait pas d'héritier masculin. Autrement dit, l'ancien rex inutilis avait trouvé un épilogue à la crise administrative mais il n'avait pas pu résoudre le problème dynastique : l'amour de Meliadice et Cleriadus sera l'unique solution envisagée et leur mariage sera justifié au niveau moral par les exploits du jeune chevalier et par la détresse de la princesse.

S'il est vrai que le traitre impose une tyrannie, elle n'est pas définie pour autant par la manière dont Thomas arrive au pouvoir mais par le fait qu'il dénonce l'élection du souverain confirmée par le conseil des barons, c'est-à-dire la désignation du comte des Asturies administrateur du royaume. Cleriadus sauve l'Angleterre et défend l'autorité légitime de son père, ainsi que le narrateur l'expose de manière elliptique au moment de la sédition contre Thomas. Le rôle du jeune chevalier impliquerait d'ailleurs que le comte des Asturies ne réussit pas complètement à préserver l'intérêt anglais parce qu'il ne peut répondre aux besoins des Anglais au détriment de ses propres vassaux ${ }^{26}$. En conséquence, l'auteur signalerait ainsi que le transfert de pouvoir à un administrateur n'est pas une solution satisfaisante: c'est uniquement le roi qui peut garantir la prospérité et l'aisance de sa nation. Qui plus est, si le gouvernant veille sur les intérêts d'une communauté, il ne peut s'occuper d'une autre, puisqu'il privilégie les besoins du pays avec lequel il établit un lien plus étroit en fonction de ses propres intérêts. Cette idée est renforcée par le narrateur lorsqu'il décrit le gouvernement de Cleriadus; il met l'accent sur le fait que le nouveau roi peut gouverner deux pays en même temps puisqu'il est la tête qui conduit les deux nations ${ }^{27}$. Cette argu-

25. Chap. XXVI, p. 346-347.

26. Il faut se rappeler qu'au moment de la crise en Angleterre, le comte des Asturies demeure dans son pays.

27. «Or dit le compte que le roy Cleriadus est en son royaume d'Angleterre où il fait, lui et Meliadice, festes, jouxtes et tous esbatemens à tous venans et se fait aymer de tous en gardent ses deux royaumes paisiblement et va souvent de l'un a l'autre. Il fait droit au pouvre comme au riche et, se ung chevalier est requis de gaige de bataille ne il n'a cheval ne harnas de quoy 
mentation, en outre, fait partie du récit et constitue un épisode du roman. En effet, la situation de l'ancien roi d'Irlande, l'oncle de Cleriadus du côté maternel, est semblable à celle de Phellipon mais, au lieu de désigner un administrateur, il abdique en faveur de son neveu, événement célébré avec magnificence à la fin du roman.

Par ailleurs, l'auteur bourguignon accroît le sens politique du roman en interpolant - de façon voilée - une affaire qui demanda l'intervention du pape Clément $\mathrm{V}$ et l'utilisation de la doctrine canonique relative à la figure du rex inutilis. L'affaire fait référence à la déposition transitoire d'Henry II de Chypre, dont le contexte aurait été évoqué dans Cleriadus et Meliadice à travers l'épisode de la guerre menée contre les Sarrasins par les Anglais et les Français à Chypre.

Pour mieux comprendre cette insertion, l'excursus suivant décrira brièvement la situation politique à Chypre pendant le XIV ${ }^{\mathrm{e}}$ siècle et les conséquences juridiques qu'elle produisit. Henry, frère de Jean I (1284-1285) de la famille Poitiers-Lusignan, reçut la couronne de Chypre, de Jérusalem et de Tyr en 1285. Le début de son règne fut caractérisé par son intérêt croissant pour la récupération d'Acre, qu'il ne parvint pas à accomplir car la ville tomba sous la domination des mamelouks. En 1291, après le désastre militaire, Henry tourna toute son attention vers Chypre, bien qu'il ait insisté pour occuper au moins une partie de la Terre Sainte, avec le roi d'Arménie Cilice et le mongol Ilkhan de Perse. Néanmoins, la guerre sainte ne fut pas la seule source de conflits pour Henry : s'y ajoutaient d'une part les grandes tensions provoquées par les Génois à cause de la guerre avec les Vénitiens et d'autre part, l'animosité génoise envers les Chypriotes à cause des bénéfices que ceux-ci donnèrent aux villes de Pise et de Barcelone. Or, si les dangers extérieurs menacèrent le monarque, le risque majeur trouva son origine au sein de sa propre famille. En effet, le frère d'Henry, Amaury, et la puissante famille rivale, les Ibelins, conspirèrent contre le roi. Le 26 avril 1306, Amaury déclara le roi incapable de régner. Les raisons invoquées se rapportaient à la mauvaise santé du roi ainsi qu'à son impéritie face aux événements politiques et sociaux du moment. À cela on pourrait ajouter la confiance accordée aux mauvais conseillers, les suggestions rejetées sur la manière de négocier avec les Génois, son incapacité à défendre le royaume du Soudan d'Égypte et l'impossibilité de collaborer avec les alliés d'Arménie et de Perse. En conséquence, Amaury prit le titre de gubernator et rector du royaume. La plupart des vassaux d'Henry acceptèrent Amaury et la prise de pouvoir se déroula sans difficulté. Il conserva sa charge jusqu'à son assassinat en 1310, lorsqu'Henry récupéra le gouvernement de Chypre.

il se puisse aider, le roy Cleriadus lui en fait bailler, et se il est exoinié de son corps que il ne puisse combatre, le roy Cleriadus lui fait bailler ung chevalier ad ce faire. Que vous diroy je ? Le roy Cleriadus et la royne Meliadice se font tant aymer de tous et de toutes, petiz et grans, et de tous leurs deux royaumes, par les grans bien qui sont en eulx, que c'est une belle chose que d'en ouyr parler. » (chap. XLV, p. 711-712). 
Ce cas fut largement divulgué en Occident. Amaury, aussi bien qu'Henry, envoyèrent leurs délégations au pape Clément V à Poitiers, et la documentation arriva jusqu'à Avignon. Les charges contre Henry II furent reproduites à partir d'une sorte de manuel sur le rex inutilis, établi par le pape Innocent IV dans sa décrétale Grandi (1245) - fondée sur le Decretum Gratiani, Alius ítem, causa 15, quæstio 6, canon $3^{28}$ - et employé pour la déposition de Sanche II de Portugal (qui fut déchu de son trône mais non pas de sa dignitas royale par son frère Alfonso en 1245). Innocent IV disposa que le roi d'une monarchie héritière ne pouvait être destitué complètement : bien au contraire, il devait conserver sa dignitas royale et avoir un curator pour conduire l'administration du royaume. Ce guvernator avait, idéalement, le droit d'accéder à la couronne en cas de décès du souverain. Des commentateurs successifs permirent l'abdication et le bannissement du roi, comme dans le cas d'Édouard II (1327) et de Richard II (1399) d'Angleterre. Tout ceci montre que le roman bourguignon établit un lien avec l'histoire récente. En effet, l'interprétation papale est presque littéralement reproduite dans Cleriadus et Meliadice: Thomas éloigne le comte des Asturies parce que l'incapacité de Phellipon permet l'éventuelle acquisition de la dignité royale par le curator. De cette manière, le comte, et même son fils Cleriadus, pourraient réclamer le trône anglais à la mort de Phellipon.

Les analogies entre les événements chypriotes et l'histoire racontée dans Cleriadus et Meliadice paraissent évidentes. Sans doute, l'auteur arrangea la thématique et l'agencement du récit afin de rappeler, chez le lecteur, le problème politique et juridique de Chypre. Les ressemblances thématiques n'étaient pourtant pas suffisamment visibles pour que l'auditoire se souvienne des faits historiques commentés préalablement et les rapporte à l'histoire de Cleriadus; de sorte que ce fait historique et toute la tradition légale furent introduits dans le récit comme s'il s'agissait de composants narratifs: l'enchaînement de la guerre à Chypre avec les mésaventures de Meliadice constituent un assemblage dont le sujet principal est en rapport avec la déposition du roi lorsque son impuissance est démontrée par les faits. En plus, le portrait du roi de Chypre dressé dans Cleriadus et Meliadice et le récit de ses problèmes avec les Sarrasins semblent faire référence aussi à l'histoire d'Henry II. Au demeurant, la figure de Phellipon et celle du roi de Chypre éclarcissent le sens que l'auteur veut donner au règne du souverain anglais : depuis la situation politique interne qui conduit à la déposition transitoire

28. Decretum Gratiani, Alius ítem, causa 15, quæstio 6, canon $3:$ «C. III. Pontificalis auctoritas a iuramento fidelitatis nonnullos absoluit. Alius item Romanus Pontifex, Zacharias scilicet regem Francorum non tam pro suis iniquitatibus, quam pro eo, quod tantae potestati erat inutilis, a regno deposuit, et Pipinum, Karoli inperatoris patrem, in eius loco substituit, omnesque Francigenas a iuramento fidelitatis absoluit. Quod etiam ex frequenti auctoritate agit sancta ecclesia, cum milites absoluit a uinculo iuramenti, quod factum est his episcopis, qui apostolica auctoritate a pontificali gradu deponuntur. » Grégoire VII utilisa aussi le canon lors de son affrontement avec l'empereur allemand Henri IV, et à la fin du XIII ${ }^{\mathrm{e}}$ siècle, il fut employé aussi pour déposer Adolphe de Nassau (1298). 
d'Henry jusqu'aux affrontements constants contre les Sarrasins pour récupérer la Terre Sainte. L'analogie entre la crise anglaise et celle de Chypre est possible, au niveau de la structure, grâce à l'imbrication de la guerre et de l'exil de Meliadice.

Dès lors, il est clair que la guerre de Cent Ans n'est pas la référence historique du roman, comme on pouvait le croire, parce que l'œuvre fut écrite au milieu du $\mathrm{XV}^{\mathrm{e}}$ siècle. Ce sont, en revanche, des faits apparamment marginaux qui sont utilisés pour dépeindre une crise de pouvoir. Il n'existe pas, cependant, d'opposition entre deux alternatives historiques puisque la situation à Chypre se référait aussi au problème de l'incapacité royale, comme en France après le règne de Charles V. Au demeurant, l'auteur fait allusion à l'affaire chypriote pour représenter la figure du rex inutilis afin d'évoquer également les difficultés politiques et sociales françaises du bas Moyen Âge.

En conclusion, la relation entre littérature, droit et histoire factuelle offre un panorama de la mentalité bourguignone $d u X V^{\mathrm{e}}$ siècle et, en même temps, nous fait comprendre que ce n'est pas le fait historique en lui-même qui a engendré le dialogue entre le texte et le contexte mais que ce sont les différentes sphères de la culture qui ont communiqué à travers certains faits historiques. Finalement, la démarche poursuivie a permis d'intégrer des éléments extra-textuels divers; on pourrait continuer en s'interrogeant sur les effets suscités par les événements sur les institutions médiévales et dans les domaines distincts de la culture (littérature, droit, philosophie, etc.), et en analysant la façon dont les phénomènes littéraires et historiques ont structuré la mentalité de cette époque.

Lidia Amor, Departamento de Investigaciones Medievales (DIMED) IMHICIHU - CONICET - Université de Buenos Aires (UBA)

\section{Droit canon et littérature chevaleresque : l'image du rex inutilis dans le roman de Cleriadus et Meliadice}

Cleriadus et Meliadice, roman chevaleresque de la première moitié du $\mathrm{XV}^{\mathrm{e}}$ siècle, raconte la biographie de Cleriadus, fils du comte des Asturies, depuis sa jeunesse jusqu'à son mariage avec Meliadice, princesse héritière du royaume anglais. Si la thématique de l'œuvre montre des rapports avec la littérature romanesque, il n'en reste pas moins qu'elle exprime une certaine appartenance du roman à la littérature édifiante, notamment en ce qui concerne les attributs et la conduite souhaités chez le prince idéal. Dans cet article, nous essayons de montrer que l'entrelacement de la fiction romanesque et de la littérature édifiante laisse percevoir une réflexion sur le rex inutilis, figure présente dans le droit canon. De telle sorte, la doctrine sur le rex inutilis devient le matériau même de la fiction et engendre un réseau mnémotechnique chez le lecteur où histoire, jurisprudence et littérature se rejoignent. 
Cleriadus et Meliadice - roman de chevalerie - motif de la dame injustement inculpée - droit canon - rex inutilis

Canon Law and Chivalric Literature : the Image of the Rex Inutilis in the Cleriadus et Meliadice Romance

Cleriadus et Meliadice, an early fifteenth-century chivalric romance, recounts the life of Cleriadus, son the count of Asturias, from his youth to his marrying princess Meliadice, heir to the kingdom of England. If the work is thematically linked to the genre of romance, it is nevertheless akin to edifying literature, particularly as far as the bearing and attitudes expected from the ideal prince are concerned. In this article, we try to show how the interlacement of romantic fiction and edifying literature can disclose a reflection about the rex inutilis, a figure whose origins are to be found in canon law. The doctrine of the rex inutilis thus becomes the very material of fiction, creating in the reader's mind a mnemonic web where history, law and literature meet.

Cleriadus et Meliadice - chivalric romance - accused women motif - canon law - rex inutilis 